\title{
Efficient synthesis of furo[2,3-d]pyrimidin-4(3H)-ones
}

\author{
Yang-Gen Hu, ${ }^{\text {a,b }}$ Gui-Hua Li, ${ }^{\text {b }}$ and Ming-Wu Ding ${ }^{\text {a, * }}$ \\ ${ }^{a}$ Key Laboratory of Pesticide \& Chemical Biology of Ministry of Education, Central China \\ Normal University, Wuhan 430079, China \\ ${ }^{\mathrm{b}}$ Department of Medicinal Chemistry, Yunyang Medical College, Shiyan 442000, China \\ E-mail: ding5229@yahoo.com.cn
}

\begin{abstract}
The carbodiimides 4 , obtained from reactions of iminophosphorane 3 with aromatic isocyanates, reacted with amines or phenols to give 2-substituted furo[2,3-d]pyrimidin-4(3H)-ones 6 in the presence of catalytic amount of sodium alkoxide or solid potassium carbonate in good yields.
\end{abstract}

Keywords: Furo[2,3-d]pyrimidin-4(3H)-one, iminophosphorane, aza-Wittig reaction, isocyanate

\section{Introduction}

The derivatives of fused pyrimidinones are valued not only for their rich and varied chemistry, but also for many important biological properties. Among them, the thienopyrimidinone ring system, because of a formal isoelectronic relationship with purine, is of special biological interest. ${ }^{1-4}$ However, there are few reports on furopyrimidinones. ${ }^{5-10}$ This is probably due to the instability of the furan ring especially under harsh reaction conditions. Therefore, the development of an efficient method for the preparation of furo[2,3-d]pyrimidinone derivatives under mild conditions is desirable. A key requirement for the facile synthesis of furo[2,3d]pyrimidinones is the use of an effective electron-withdrawing group in order to protect the electron-rich furans from polymerization reactions. The ester group is a very useful electronwithdrawing protecting group since carboxylic ester furans are relatively easy to prepare and are extremely stable. Such protection allows for further functionalization of the furan ring.

The aza-Wittig reactions of iminophosphoranes have received increased attention in view of their utility in the synthesis of nitrogen heterocyclic compounds under mild conditions. ${ }^{11-16}$ Recently we have been interested in the synthesis of quinazolinones, thienopyrimidinones and imidazolinones via aza-Wittig reaction. ${ }^{17-21}$ Here we wish to report an efficient approach to the synthesis of furo[2,3-d]pyrimidin-4(3H)-ones under mild conditions. 


\section{Results and Discussion}

Diethyl 2-amino-5-methylfuran-3,4-dicarboxylate 2 was prepared from reaction of ethyl 2chloroacetoacetate 1 with ethyl cyanoacetate in the presence of sodium ethoxide in only 58\% yield according to literature report ${ }^{22}$. However, we found that when triethylamine was used as a base in place of sodium ethoxide, compound 2 was obtained in $88 \%$ yield. The good yield may be due to the mild conditions as weak base triethylamine was used. Compound 2 was further converted to iminophosphorane 3 via reaction with triphenylphosphine, hexachloroethane and triethylamine in good yield (Scheme 1).

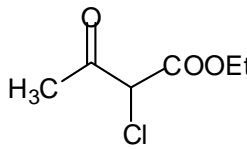

1
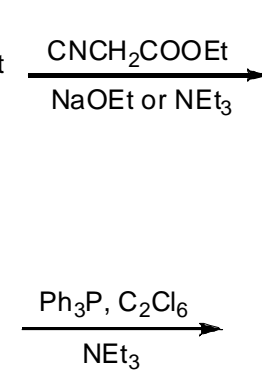

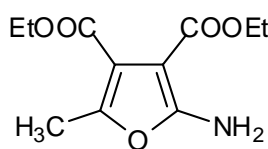

2

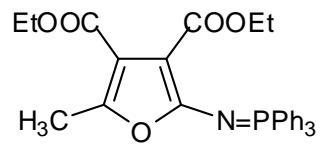

3

\section{Scheme 1}

Iminophosphorane 3 reacted with aromatic isocyanates at $0-5{ }^{\circ} \mathrm{C}$ to give carbodiimides $\mathbf{4}$, which were allowed to react with secondary amines to provide guanidine intermediates $\mathbf{5}$. In the presence of catalytic amount of sodium ethoxide, the intermediates 5 were converted easily to 2dialkylamino furo[2,3-d]pyrimidin-4(3H)-ones $\mathbf{6 a - h}$ in satisfactory yields at room temperature (Scheme 2). The reaction of carbodiimide 4 with phenols in the presence of a catalytic amount of anhydrous potassium carbonate produces 2-aryloxyfuro[2,3- $d$ ]pyrimidin-4(3H)-ones 6i-o in good yields at $50-60^{\circ} \mathrm{C}$. No matter whether the substituents on the phenols are electron-withdrawing or electron-releasing groups, the cyclization can be completed smoothly under mild conditions. The results are listed in Table 1.

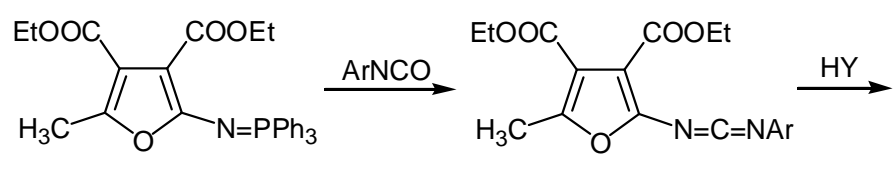

3

4

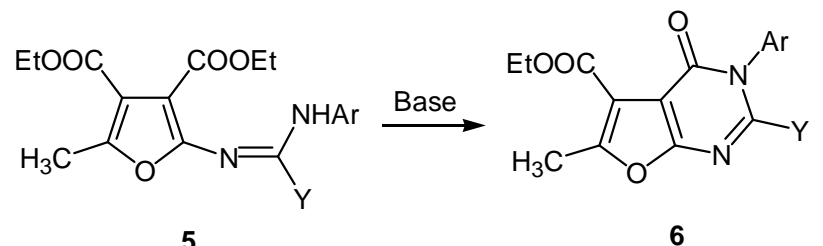

\section{Scheme 2}


Table1. Synthesis of Compounds 6

\begin{tabular}{|c|c|c|c|}
\hline Compd & $\mathrm{Ar}$ & $\mathrm{Y}$ & Yield $(\%)^{a}$ \\
\hline $6 \mathbf{a}$ & $\mathrm{Ph}$ & $\mathrm{NEt}_{2}$ & 85 \\
\hline 6b & $\mathrm{Ph}$ & piperidin-1-yl & 88 \\
\hline $6 c$ & $\mathrm{Ph}$ & morpholin-4-yl & 86 \\
\hline 6d & $3-\mathrm{MeC}_{6} \mathrm{H}_{4}$ & piperidin-1-yl & 90 \\
\hline $6 e$ & $4-\mathrm{ClC}_{6} \mathrm{H}_{4}$ & $\mathrm{NEt}_{2}$ & 86 \\
\hline $6 f$ & $4-\mathrm{ClC}_{6} \mathrm{H}_{4}$ & morpholin-4-yl & 87 \\
\hline $6 g$ & $4-\mathrm{FC}_{6} \mathrm{H}_{4}$ & $\mathrm{NEt}_{2}$ & 85 \\
\hline $6 \mathrm{~h}$ & $4-\mathrm{FC}_{6} \mathrm{H}_{4}$ & morpholin-4-yl & 83 \\
\hline $6 \mathbf{i}$ & $\mathrm{Ph}$ & 4- $\mathrm{MeOC}_{6} \mathrm{H}_{4} \mathrm{O}$ & 89 \\
\hline $6 \mathbf{j}$ & $\mathrm{Ph}$ & $3-\mathrm{MeC}_{6} \mathrm{H}_{4} \mathrm{O}$ & 87 \\
\hline $6 \mathbf{k}$ & $\mathrm{Ph}$ & 2- $\mathrm{MeC}_{6} \mathrm{H}_{4} \mathrm{O}$ & 87 \\
\hline $6 \mathbf{I}$ & $\mathrm{Ph}$ & $3-\mathrm{NO}_{2} \mathrm{C}_{6} \mathrm{H}_{4} \mathrm{O}$ & 86 \\
\hline $6 m$ & $\mathrm{Ph}$ & $2,4-2 \mathrm{ClC}_{6} \mathrm{H}_{3} \mathrm{O}$ & 84 \\
\hline $6 n$ & $3-\mathrm{MeC}_{6} \mathrm{H}_{4}$ & $3-\mathrm{NO}_{2} \mathrm{C}_{6} \mathrm{H}_{4} \mathrm{O}$ & 87 \\
\hline 60 & $3-\mathrm{MeC}_{6} \mathrm{H}_{4}$ & 2-naphthaloxy & 79 \\
\hline
\end{tabular}

${ }^{a}$ Isolated yields based on iminophosphorane 3 .

The structures of compound 6 were established based on their NMR, MS, IR and elementary analysis. For example the ${ }^{1} \mathrm{H}$ NMR spectral data of $\mathbf{6 a}$ show the signals of $-\mathrm{OCH}_{2}$ or $-\mathrm{NCH}_{2}$ at 4.36 or $3.13 \mathrm{ppm}$ as quartets, signals of $\mathrm{CH}_{3}$ at $2.62,1.37$ or $0.86 \mathrm{ppm}$ as singlet or triplets. The phenyl signals appeared at 7.46-7.28 ppm. In the IR spectrum of compound $\mathbf{6 a}$, the strong stretching vibration peak of two $\mathrm{C}=\mathrm{O}$ appears at $1707 \mathrm{~cm}^{-1}$. The MS spectrum of $6 \mathbf{a}$ shows molecule ion peak $\left(\mathrm{M}^{+}\right)$at $\mathrm{m} / \mathrm{z} 369$ with $100 \%$ abundance.

In conclusion, we have developed an efficient synthesis of various substituted furo[2,3d]pyrimidin-4(3H)-ones in good yield via aza-Wittig reaction of an iminophosphorane. Due to the easily accessible and versatile starting material, this method has the potential in synthesis of many biologically and pharmaceutically active furopyrimidinone derivatives.

\section{Experimental Section}

General Procedures. Melting points are uncorrected. MS were measured on a Finnigan Trace MS spectrometer. IR spectra were recorded on a PE-983 infrared spectrometer as $\mathrm{KBr}$ pellets with absorption in $\mathrm{cm}^{-1}$. NMR spectra were recorded in $\mathrm{CDCl}_{3}$ on a Varian Mercury 400 spectrometer and resonances are given in ppm $(\delta)$ relative to TMS. Elementary analyses were taken on a Vario EL III elementary analysis instrument. 
Preparation of diethyl 2-amino-5-methylfuran-3,4-dicarboxylate (2). To a mixture of ethyl cyanoacetate $(3.39 \mathrm{~g}, 30 \mathrm{mmol})$ and $\mathrm{NEt}_{3}(8 \mathrm{~mL}, 56 \mathrm{mmol})$ in $\mathrm{EtOH}(15 \mathrm{~mL})$ was added dropwise ethyl 2-chloroacetoacetate $1(4.93 \mathrm{~g}, 30 \mathrm{mmol})$ at room temperature. After stirring for $1 \mathrm{~h}$, the solvent was removed under reduced pressure and the residue was recrystallized from EtOH/ $\mathrm{H}_{2} \mathrm{O}(1: 1)$ to give diethyl 2-amino-5-methylfuran-3,4-dicarboxylate 2 . White solid (6.34 g, yield $88 \%$ ), m.p. $85-86^{\circ} \mathrm{C}$, lit. ${ }^{22}$ m.p. $81.5-82.5{ }^{\circ} \mathrm{C}$.

Preparation of $\mathbf{N}$-(3,4-diethoxycarbonyl-5-methylfuran-2-yl)iminotriphenylphosphorane (3). To a mixture of diethyl 2-amino-5-methylfuran-3,4-dicarboxylate 2 (2.41 g, $10 \mathrm{mmol}, \mathrm{PPh}_{3}$ (3.93 g, $15 \mathrm{mmol})$ and $\mathrm{C}_{2} \mathrm{Cl}_{6}(3.56 \mathrm{~g}, 15 \mathrm{mmol})$ in dry $\mathrm{CH}_{3} \mathrm{CN}(30 \mathrm{~mL})$, was added dropwise $\mathrm{NEt}_{3}(4.2 \mathrm{~mL}, 30 \mathrm{mmol})$ at room temperature. After stirred for 1-2 $\mathrm{h}$, the solvent was removed under reduced pressure and the residue was recrystallized from $\mathrm{EtOH}$ to give iminophosphorane 3 as white solid (4.06 g, yield 81\%), m.p. $169-170{ }^{\circ} \mathrm{C} .{ }^{1} \mathrm{H} \mathrm{NMR}\left(\mathrm{CDCl}_{3}, 400 \mathrm{MHz}\right): \delta 7.82-7.45$ (m, 15H, Ar-H), 4.28-4.20 (m, 4H, 2OCH $), 2.62\left(\mathrm{~s}, 3 \mathrm{H}, \mathrm{CH}_{3}\right), 1.28\left(\mathrm{t}, J=7.2 \mathrm{~Hz}, 6 \mathrm{H}, 2 \mathrm{CH}_{3}\right)$. IR (KBr): $1727(\mathrm{C}=\mathrm{O}), 1681,1562,1109 \mathrm{~cm}^{-1} . \mathrm{MS} \mathrm{m} / \mathrm{z}(\%): 501$ (100, $\left.\mathrm{M}^{+}\right), 456$ (66), 260 (98), 182 (98), 107 (92), 77 (69). Anal. Calcd for $\mathrm{C}_{29} \mathrm{H}_{28} \mathrm{NO}_{5} \mathrm{P}$ (501.5): C, 69.45; H, 5.63; N, 2.79. Found: C, 69.22, H, 5.73; N, 2.95.

\section{General preparation of 2-dialkylaminofuro[2,3-d]pyrimidines 6a-6h}

To a solution of iminophosphorane $3(1.0 \mathrm{~g}, 2 \mathrm{mmol})$ in dry methylene chloride $(15 \mathrm{~mL})$ was added aromatic isocyanate $(2 \mathrm{mmol})$ under nitrogen at room temperature. After the reaction mixture was stood for 8-12 hours at $0-5{ }^{\circ} \mathrm{C}$, the solvent was removed off under reduced pressure and ether/petroleum ether $(1: 2,20 \mathrm{~mL})$ was added to precipitate triphenylphosphine oxide. After filtration the solvent was removed to give carbodiimide 4 , which was used directly without further purification. To the solution of 4 prepared above in methylene chloride $(15 \mathrm{ml})$ was added dialkylamine $(2 \mathrm{mmol})$. After the reaction mixture was allowed to stand for $0.5-6 \mathrm{~h}$, the solution was condensed and anhyd. EtOH $(8 \mathrm{~mL})$ with EtONa $(0.3 \mathrm{mmol}, 10 \%$ equiv) in EtOH was added. The mixture was stirred for 4-6 h at $\mathrm{r}$. t. The solution was condensed and the residue was recrystallized from EtOH to give 6a-6h.

2-(Diethylamino)-5-ethoxycarbonyl-6-methyl-3-phenylfuro[2,3-d]pyrimidin-4(3H)-one (6a). White crystals (yield 0.63 g, 85\%), m.p. 160-161 ${ }^{\circ} \mathrm{C}$. IR (KBr): 1707 (C=O), 1589, 1538, $1233 \mathrm{~cm}^{-1} .{ }^{1} \mathrm{H}$ NMR $\left(400 \mathrm{MHz}, \mathrm{CDCl}_{3}\right): \delta 7.46-7.28(\mathrm{~m}, 5 \mathrm{H}, \mathrm{Ar}-\mathrm{H}), 4.36(\mathrm{q}, J=7.2 \mathrm{~Hz}, 2 \mathrm{H}$, $\mathrm{OCH}_{2}$ ), 3.13 (q, $\left.J=7.2 \mathrm{~Hz}, 4 \mathrm{H}, 2 \times \mathrm{NCH}_{2}\right), 2.62\left(\mathrm{~s}, 3 \mathrm{H}, \mathrm{CH}_{3}\right), 1.37$ (t, $\left.J=7.2 \mathrm{~Hz}, 3 \mathrm{H}, \mathrm{CH}_{3}\right), 0.86$ (t, $\left.J=7.2 \mathrm{~Hz}, 6 \mathrm{H}, 2 \times \mathrm{CH}_{3}\right)$. MS (m/z, \%): $369\left(\mathrm{M}^{+}, 100\right), 323$ (27), 295 (26), 175 (72), 118 (47), 105 (56). Anal. Calcd for $\mathrm{C}_{20} \mathrm{H}_{23} \mathrm{~N}_{3} \mathrm{O}_{4}$ (369.4): C, 65.03; H, 6.28; N, 11.37. Found: C, 65.20, H, $6.53 ; \mathrm{N}, 11.34$.

\section{5-Ethoxycarbonyl-6-methyl-3-phenyl-2-(piperidin-1-yl)furo[2,3-d]pyrimidin-4(3H)-one}

(6b). White crystals (yield 0.67 g, 88\%), m.p. 153-155 ${ }^{\circ} \mathrm{C}$. IR (KBr): $1705(\mathrm{C}=\mathrm{O}), 1534$, $1250 \mathrm{~cm}^{-1} .{ }^{1} \mathrm{H}$ NMR $\left(400 \mathrm{MHz}, \mathrm{CDCl}_{3}\right): \delta 7.47-7.31(\mathrm{~m}, 5 \mathrm{H}, \mathrm{Ar}-\mathrm{H}), 4.36(\mathrm{q}, J=7.2 \mathrm{~Hz}, 2 \mathrm{H}$, $\left.\mathrm{OCH}_{2}\right), 3.12\left(\mathrm{q}, J=5.2 \mathrm{~Hz}, 4 \mathrm{H}, 2 \times \mathrm{NCH}_{2}\right), 2.62\left(\mathrm{~s}, 3 \mathrm{H}, \mathrm{CH}_{3}\right), 1.37\left(\mathrm{t}, J=7.2 \mathrm{~Hz}, 3 \mathrm{H}, \mathrm{CH}_{3}\right), 1.34-$ 
1.25 (m, 6H, 3× $\left.\mathrm{CH}_{2}\right)$. MS (m/z, \%): $381\left(\mathrm{M}^{+}, 100\right), 335$ (36), 307 (27), 187 (52), 159 (11). Anal. Calcd for $\mathrm{C}_{21} \mathrm{H}_{23} \mathrm{~N}_{3} \mathrm{O}_{4}$ (381.4): C, 66.13; H, 6.08; N, 11.02. Found: C, 66.17, H, 6.23; N, 10.74. 5-Ethoxycarbonyl-6-methyl-2-(morpholin-4-yl)-3-phenylfuro[2,3-d]pyrimidin-4(3H)-one (6c). White crystals (yield 0.66 g, 86\%), m.p. $174-175^{\circ} \mathrm{C}$. IR (KBr): $1714(\mathrm{C}=\mathrm{O}), 1589,1541$, $1114 \mathrm{~cm}^{-1} .{ }^{1} \mathrm{H}$ NMR $\left(400 \mathrm{MHz}, \mathrm{CDCl}_{3}\right): \delta 7.51-7.27(\mathrm{~m}, 5 \mathrm{H}, \mathrm{Ar}-\mathrm{H}), 4.38(\mathrm{q}, J=7.2 \mathrm{~Hz}, 2 \mathrm{H}$, $\left.\mathrm{OCH}_{2}\right), 3.44\left(\mathrm{t}, J=4.8 \mathrm{~Hz}, 4 \mathrm{H}, 2 \times \mathrm{OCH}_{2}\right), 3.14\left(\mathrm{t}, J=4.8 \mathrm{~Hz}, 4 \mathrm{H}, 2 \times \mathrm{NCH}_{2}\right), 2.64\left(\mathrm{~s}, 3 \mathrm{H}, \mathrm{CH}_{3}\right)$, 1.38 (t, $\left.J=7.2 \mathrm{~Hz}, 3 \mathrm{H}, \mathrm{CH}_{3}\right)$. MS (m/z, \%): $383\left(\mathrm{M}^{+}, 100\right), 337$ (72), 309 (68), 134 (30), 123 (29). Anal. Calcd for $\mathrm{C}_{20} \mathrm{H}_{21} \mathrm{~N}_{3} \mathrm{O}_{5}$ (383.4): C, 62.65; H, 5.52; N, 10.96. Found: C, 62.38, H, 5.56; $\mathrm{N}, 10.88$.

\section{5-Ethoxycarbonyl-6-methyl-3-(3-methylphenyl)-2-(piperidin-1-yl)furo[2,3-d]pyrimidin-}

4(3H)-one (6d). White crystals (yield $0.71 \mathrm{~g}, 90 \%$ ), m.p. $158-159{ }^{\circ} \mathrm{C}$. IR (KBr): $1705(\mathrm{C}=\mathrm{O})$, 1591, 1534, 1372, $1099 \mathrm{~cm}^{-1} .{ }^{1} \mathrm{H}$ NMR (400 MHz, $\left.\mathrm{CDCl}_{3}\right): \delta$ 7.36-7.10 (m, 4H, Ar-H), 4.38 (q, $J$ $\left.=7.2 \mathrm{~Hz}, 2 \mathrm{H}, \mathrm{OCH}_{2}\right), 3.13\left(\mathrm{t}, J=4.8 \mathrm{~Hz}, 4 \mathrm{H}, 2 \times \mathrm{NCH}_{2}\right), 2.64\left(\mathrm{~s}, 3 \mathrm{H}, \mathrm{CH}_{3}\right), 2.60\left(\mathrm{~s}, 3 \mathrm{H}, \mathrm{Ar}-\mathrm{CH}_{3}\right)$, 1.46-1.22 (m, 6H, $\left.3 \times \mathrm{CH}_{2}\right), 1.38\left(\mathrm{t}, \mathrm{J}=7.2 \mathrm{~Hz}, 3 \mathrm{H}, \mathrm{CH}_{3}\right) .{ }^{13} \mathrm{C} \mathrm{NMR}\left(100 \mathrm{MHz}, \mathrm{CDCl}_{3}\right): \delta 13.6$, 14.3, 21.2, 23.9, 24.8, 49.8, 60.7, 98.1, 111.0, 125.8, 128.6, 128.7, 129.4, 137.8, 138.6, 155.9, 157.4, 158.2, 163.1, 163.7. MS (m/z, \%): $395\left(\mathrm{M}^{+}, 48\right), 350$ (11), 320 (15), 201 (100), 174 (51), 145 (34), 91 (68). Anal. Calcd for $\mathrm{C}_{22} \mathrm{H}_{25} \mathrm{~N}_{3} \mathrm{O}_{4}$ (395.5): C, 66.82; H, 6.37; N, 10.63. Found: C, $66.85, \mathrm{H}, 6.40 ; \mathrm{N}, 10.50$.

\section{3-(4-Chlorophenyl)-2-(diethylamino)-5-ethoxycarbonyl-6-methylfuro[2,3-d]pyrimidin-}

4(3H)-one (6e). White crystals (yield 0.66 g, 82\%), m.p. $170-172{ }^{\circ} \mathrm{C} . \mathrm{IR}(\mathrm{KBr}): 1712(\mathrm{C}=\mathrm{O})$, 1589, 1532, $1114 \mathrm{~cm}^{-1} .{ }^{1} \mathrm{HNMR}\left(400 \mathrm{MHz}, \mathrm{CDCl}_{3}\right): \delta 7.46-7.23(\mathrm{~m}, 4 \mathrm{H}, \mathrm{Ar}-\mathrm{H}), 4.38(\mathrm{q}, J=7.2$ $\left.\mathrm{Hz}, 2 \mathrm{H}, \mathrm{OCH}_{2}\right), 3.13\left(\mathrm{q}, J=7.2 \mathrm{~Hz}, 4 \mathrm{H}, 2 \times \mathrm{NCH}_{2}\right), 2.62\left(\mathrm{~s}, 3 \mathrm{H}, \mathrm{CH}_{3}\right), 1.38(\mathrm{t}, J=7.2 \mathrm{~Hz}, 3 \mathrm{H}$, $\left.\mathrm{CH}_{3}\right), 0.90\left(\mathrm{t}, J=7.2 \mathrm{~Hz}, 6 \mathrm{H}, 2 \times \mathrm{CH}_{3}\right) . \mathrm{MS}(\mathrm{m} / \mathrm{z}, \%): 403\left(\mathrm{M}^{+}, 42\right), 357$ (20), 329 (17), 209 (100), 179 (32), 153 (25), 138 (44), 111 (32). Anal. Calcd for $\mathrm{C}_{20} \mathrm{H}_{22} \mathrm{ClN}_{3} \mathrm{O}_{4}$ (403.9): C, 59.48; H, 5.49; N, 10.40. Found: C, 59.54, H, 5.73; N, 10.24.

\section{3-(4-Chlorophenyl)-5-ethoxycarbonyl-6-methyl-2-(morpholin-4-yl)furo[2,3-d]pyrimidin-} 4(3H)-one (6f). White crystals (yield 0.73 g, 87\%), m.p. $158-160{ }^{\circ} \mathrm{C}$. IR (KBr): $1705(\mathrm{C}=\mathrm{O})$, 1689, 1538, $1245 \mathrm{~cm}^{-1} .{ }^{1} \mathrm{H}$ NMR (400 MHz, $\left.\mathrm{CDCl}_{3}\right): \delta 7.48-7.27(\mathrm{~m}, 4 \mathrm{H}, \mathrm{Ar}-\mathrm{H}), 4.38(\mathrm{q}, J=7.2$ $\left.\mathrm{Hz}, 2 \mathrm{H}, \mathrm{OCH}_{2}\right), 3.48\left(\mathrm{t}, J=4.8 \mathrm{~Hz}, 4 \mathrm{H}, 2 \times \mathrm{OCH}_{2}\right), 3.15\left(\mathrm{t}, J=4.8 \mathrm{~Hz}, 4 \mathrm{H}, 2 \times \mathrm{NCH}_{2}\right), 2.63(\mathrm{~s}$, $\left.3 \mathrm{H}, \mathrm{CH}_{3}\right), 1.38\left(\mathrm{t}, J=7.2 \mathrm{~Hz}, 3 \mathrm{H}, \mathrm{CH}_{3}\right)$. MS (m/z, \%): $417\left(\mathrm{M}^{+}, 100\right), 371$ (58), 343 (41), 297 (32), 223 (63), 179 (15), 167 (10). Anal. Calcd for $\mathrm{C}_{20} \mathrm{H}_{20} \mathrm{ClN}_{3} \mathrm{O}_{5}$ (417.8): C, 57.49; H, 4.82; N, 10.06. Found: C, 57.64, H, 4.63; N, 10.11 .

\section{2-(Diethylamino)-5-ethoxycarbonyl-3-(4-fluorophenyl)-6-methylfuro[2,3-d]pyrimidin-}

4(3H)-one (6g). White crystals (yield 0.66 g, 85\%), m.p. 131-132 ${ }^{\circ} \mathrm{C}$. IR (KBr): $1704(\mathrm{C}=\mathrm{O})$, 1689, 1545, $1233 \mathrm{~cm}^{-1} .{ }^{1} \mathrm{HNMR}\left(400 \mathrm{MHz}, \mathrm{CDCl}_{3}\right): \delta 7.29-7.14(\mathrm{~m}, 4 \mathrm{H}, \mathrm{Ar}-\mathrm{H}), 4.38(\mathrm{q}, J=7.2$ $\left.\mathrm{Hz}, 2 \mathrm{H}, \mathrm{OCH}_{2}\right), 3.13\left(\mathrm{t}, J=7.2 \mathrm{~Hz}, 4 \mathrm{H}, 2 \times \mathrm{NCH}_{2}\right), 2.63\left(\mathrm{~s}, 3 \mathrm{H}, \mathrm{CH}_{3}\right), 1.38(\mathrm{t}, J=7.2 \mathrm{~Hz}, 3 \mathrm{H}$, $\left.\mathrm{CH}_{3}\right), 0.89\left(\mathrm{t}, J=7.2 \mathrm{~Hz}, 6 \mathrm{H}, 2 \times \mathrm{CH}_{3}\right) .{ }^{13} \mathrm{C} \mathrm{NMR}\left(100 \mathrm{MHz}, \mathrm{CDCl}_{3}\right): \delta 12.4,13.7,14.3,45.2$, $60.8,110.9,115.9,116.2,130.7,130.8,133.9,156.1,157.0,158.4,163.1,163.9 . \mathrm{MS}(\mathrm{m} / \mathrm{z}, \%)$ : $387\left(\mathrm{M}^{+}, 62\right), 341$ (21), 313 (19), 287 (16), 193 (100), 168 (68), 137 (76), 123 (75). Anal. Calcd for $\mathrm{C}_{20} \mathrm{H}_{22} \mathrm{FN}_{3} \mathrm{O}_{4}$ (387.4): C, 62.01; H, 5.72; N, 10.85. Found: C, 62.25, H, 5.66; N, 10.82 . 
5-Ethoxycarbonyl-3-(4-fluorophenyl)-6-methyl-2-(morpholin-4-yl)furo[2,3-d]pyrimidin4(3H)-one (6h). White crystals (yield 0.67 g, 85 \%), m.p. 169-170 ${ }^{\circ} \mathrm{C}$. IR $(\mathrm{KBr}): 1714(\mathrm{C}=\mathrm{O})$, $1580,1541,1216 \mathrm{~cm}^{-1} .{ }^{1} \mathrm{H}$ NMR $\left(400 \mathrm{MHz}, \mathrm{CDCl}_{3}\right): \delta 7.34-7.16(\mathrm{~m}, 4 \mathrm{H}, \mathrm{Ar}-\mathrm{H}), 4.38(\mathrm{q}, J=7.2$ $\left.\mathrm{Hz}, 2 \mathrm{H}, \mathrm{OCH}_{2}\right), 3.48\left(\mathrm{t}, J=4.8 \mathrm{~Hz}, 4 \mathrm{H}, 2 \times \mathrm{OCH}_{2}\right), 3.14\left(\mathrm{t}, J=4.8 \mathrm{~Hz}, 4 \mathrm{H}, 2 \times \mathrm{NCH}_{2}\right), 2.63(\mathrm{~s}$, $\left.3 \mathrm{H}, \mathrm{CH}_{3}\right), 1.38\left(\mathrm{t}, J=7.2 \mathrm{~Hz}, 3 \mathrm{H}, \mathrm{CH}_{3}\right) . \mathrm{MS}(\mathrm{m} / \mathrm{z}, \%): 401\left(\mathrm{M}^{+}, 96\right), 355$ (69), $327(64), 207$ (100), 163 (74), 134 (40), 122 (33). Anal. Calcd for $\mathrm{C}_{20} \mathrm{H}_{20} \mathrm{FN}_{3} \mathrm{O}_{5}$ (401.4): C, 59.85; H, 5.02; N, 10.47. Found: C, 59.68, H, 5.26; N, 10.33.

\section{General Preparation of 2-aryloxy furo[2,3-d]pyrimidines 6i-6o}

To the solution of carbodiimide 4 (ca. $3 \mathrm{mmol}$ ) prepared above in $\mathrm{CH}_{3} \mathrm{CN}$ (15 mL) was added $\mathrm{K}_{2} \mathrm{CO}_{3}(0.03 \mathrm{~g}, 0.2 \mathrm{mmol})$ and $\mathrm{ArOH}(3 \mathrm{mmol})$ in anhydrous $\mathrm{CH}_{3} \mathrm{CN}(10 \mathrm{~mL})$. The mixture was stirred for $6-8 \mathrm{~h}$ at $50-60{ }^{\circ} \mathrm{C}$. The solution was concentrated under reduced pressure and the residual was recrystallized from ethanol/dichloromethane $(1: 2, \mathrm{v} / \mathrm{v})$ to give $\mathbf{6 i - 6 o}$.

5-Ethoxycarbonyl-2-(4-methoxyphenoxy)-6-methyl-3-phenylfuro[2,3-d]pyrimidin-4(3H)one (6i). White crystals (yield 0.75 g, 89\%), m.p. 159-161 ${ }^{\circ} \mathrm{C}$. IR (KBr): $1732(\mathrm{C}=\mathrm{O}), 1687$, 1554, $1200 \mathrm{~cm}^{-1} .{ }^{1} \mathrm{H}$ NMR (400 MHz, $\left.\mathrm{CDCl}_{3}\right): \delta$ 7.53-6.86 (m, 9H, Ar-H), 4.33 (q, J=7.2 Hz, $\left.2 \mathrm{H}, \mathrm{OCH}_{2}\right), 3.78\left(\mathrm{~s}, 3 \mathrm{H}, \mathrm{OCH}_{3}\right), 2.59\left(\mathrm{~s}, 3 \mathrm{H}, \mathrm{CH}_{3}\right), 1.34$ (t, J=7.2 Hz, 3H, $\left.\mathrm{CH}_{3}\right) . \mathrm{MS}(\mathrm{m} / \mathrm{z}, \%)$ : $420\left(\mathrm{M}^{+}, 100\right), 374$ (28), 297 (91), 268 (58), 155 (21), 145 (41). Anal. Calcd for $\mathrm{C}_{23} \mathrm{H}_{20} \mathrm{~N}_{2} \mathrm{O}_{6}$ (420.4): C, 65.71; H, 4.79; N, 6.66. Found: C, 65.85, H, 4.83; N, 6.38.

\section{5-Ethoxycarbonyl-6-methyl-2-(3-methylphenoxy)-3-phenylfuro[2,3-d]pyrimidin-4(3H)-one}

(6j). White crystals (yield 0.70 g, 87\%), m.p. 181-183 ${ }^{\circ} \mathrm{C}$. IR (KBr): $1732(\mathrm{C}=\mathrm{O}), 1688,1554$, $1205 \mathrm{~cm}^{-1} .{ }^{1} \mathrm{H}$ NMR $\left(400 \mathrm{MHz}, \mathrm{CDCl}_{3}\right): \delta$ 7.54-6.96 (m, 9H, Ar-H), $4.37(\mathrm{q}, J=7.2 \mathrm{~Hz}, 2 \mathrm{H}$, $\left.\mathrm{OCH}_{2}\right), 2.60\left(\mathrm{~s}, 3 \mathrm{H}, \mathrm{CH}_{3}\right), 2.34\left(\mathrm{~s}, 3 \mathrm{H}, \mathrm{CH}_{3}\right), 1.38$ (t, J=7.2 Hz, 3H, $\left.\mathrm{CH}_{3}\right) . \mathrm{MS}(\mathrm{m} / \mathrm{z}, \%): 404$ $\left(\mathrm{M}^{+}, 96\right), 358$ (80), 297 (100), 268 (58), 198 (33), 145 (76). Anal. Calcd for $\mathrm{C}_{23} \mathrm{H}_{20} \mathrm{~N}_{2} \mathrm{O}_{5}(404.4)$ : C, 68.31; H, 4.98; N, 6.93. Found: C, 68.27, H, 5.12; N, 6.68.

\section{5-Ethoxycarbonyl-6-methyl-2-(2-methylphenoxy)-3-phenylfuro[2,3-d]pyrimidin-4(3H)-one}

(6k). White crystals (yield 0.70 g, 87\%), m.p. 196-198 ${ }^{\circ} \mathrm{C}$. IR (KBr): $1732(\mathrm{C}=\mathrm{O}), 1689(\mathrm{C}=\mathrm{O})$, 1554, $1238 \mathrm{~cm}^{-1} .{ }^{1} \mathrm{H}$ NMR (400 MHz, $\left.\mathrm{CDCl}_{3}\right): \delta 7.57-7.05(\mathrm{~m}, 9 \mathrm{H}, \mathrm{Ar}-\mathrm{H}), 4.39$ (q, J = 7.2 Hz, 2H, $\left.\mathrm{OCH}_{2}\right), 2.60\left(\mathrm{~s}, 3 \mathrm{H}, \mathrm{CH}_{3}\right), 2.10\left(\mathrm{~s}, 3 \mathrm{H}, \mathrm{CH}_{3}\right), 1.38\left(\mathrm{t}, J=7.2 \mathrm{~Hz}, 3 \mathrm{H}, \mathrm{CH}_{3}\right) . \mathrm{MS}(\mathrm{m} / \mathrm{z}, \%)$ : $404\left(\mathrm{M}^{+}, 23\right), 358$ (19), 297 (100), 269 (91), 198 (27), 145 (83). Anal. Calcd for $\mathrm{C}_{23} \mathrm{H}_{20} \mathrm{~N}_{2} \mathrm{O}_{5}$ (404.4): C, 68.31; H, 4.98; N, 6.93. Found: C, 68.15, H, 4.91; N, 6.85.

\section{5-Ethoxycarbonyl-6-methyl-2-(3-nitrophenoxy)-3-phenylfuro[2,3-d]pyrimidin-4(3H)-one} (6l). White crystals (yield 0.75 g, 86\%), m.p. 204-206 ${ }^{\circ} \mathrm{C}$. IR (KBr): $1734(\mathrm{C}=\mathrm{O}), 1695(\mathrm{C}=\mathrm{O})$, 1554, $1217 \mathrm{~cm}^{-1} .{ }^{1} \mathrm{H}$ NMR (400 MHz, $\left.\mathrm{CDCl}_{3}\right): \delta$ 8.16-7.36 (m, 9H, Ar-H), 4.39 (q, J=7.2 Hz, $\left.2 \mathrm{H}, \mathrm{OCH}_{2}\right), 2.63\left(\mathrm{~s}, 3 \mathrm{H}, \mathrm{CH}_{3}\right), 1.38\left(\mathrm{t}, J=7.2 \mathrm{~Hz}, 3 \mathrm{H}, \mathrm{CH}_{3}\right) . \mathrm{MS}(\mathrm{m} / \mathrm{z}, \%): 435\left(\mathrm{M}^{+}, 17\right), 389$ (30), 297 (58), 269 (100), 241 (83), 198 (22), 145 (76), 118 (34). Anal. Calcd for $\mathrm{C}_{22} \mathrm{H}_{17} \mathrm{~N}_{3} \mathrm{O}_{7}$ (435.4): C, 60.69; H, 3.94; N, 9.65. Found: C, 60.41, H, 3.79; N, 9.78.

2-(2,4-Dichlorophenoxy)-5-ethoxycarbonyl-6-methyl-3-phenylfuro[2,3-d]pyrimidin-4(3H)one (6m). White crystals (yield 0.77 g, 84\%), m.p. 161-162 ${ }^{\circ} \mathrm{C}$. IR (KBr): $1727(\mathrm{C}=\mathrm{O}), 1699$ $(\mathrm{C}=\mathrm{O}), 1555,1295 \mathrm{~cm}^{-1} .{ }^{1} \mathrm{H}$ NMR $\left(400 \mathrm{MHz}, \mathrm{CDCl}_{3}\right): \delta$ 7.57-7.12 (m, 8H, Ar-H), $4.38(\mathrm{q}, J=$ 
$\left.7.2 \mathrm{~Hz}, 2 \mathrm{H}, \mathrm{OCH}_{2}\right), 2.64\left(\mathrm{~s}, 3 \mathrm{H}, \mathrm{CH}_{3}\right), 1.38\left(\mathrm{t}, J=7.2 \mathrm{~Hz}, 3 \mathrm{H}, \mathrm{CH}_{3}\right) .{ }^{13} \mathrm{C} \mathrm{NMR}(100 \mathrm{MHz}$, $\left.\mathrm{CDCl}_{3}\right): \delta 13.6,14.3,60.9,100.6,111.1,124.5,127.6,128.2,129.3,129.5,130.3,132.6,134.4$, 145.9, 154.1, 156.7, 157.5, 162.1, 162.7. MS (m/z, \%): $458\left(\mathrm{M}^{+}, 44\right), 414$ (37), 297 (100), 269 (73), 194 (25), 145 (68), 118 (24). Anal. Calcd for $\mathrm{C}_{22} \mathrm{H}_{16} \mathrm{Cl}_{2} \mathrm{~N}_{2} \mathrm{O}_{5}$ (459.3): C, 57.53; H, 3.51; N, 6.10. Found: C, 57.47, H, 3.48; N, 6.32 .

5-Ethoxycarbonyl-6-methyl-3-(3-methylphenyl)-2-(3-nitrophenoxy)furo[2,3-d]pyrimidin4(3H)-one (6n). White crystals (yield 0.78 g, 87\%), m.p. 201-202 ${ }^{\circ} \mathrm{C}$. IR (KBr): $1732(\mathrm{C}=\mathrm{O})$, $1700,1552,1296 \mathrm{~cm}^{-1} .{ }^{1} \mathrm{H}$ NMR $\left(400 \mathrm{MHz}, \mathrm{CDCl}_{3}\right): \delta 8.16-7.16(\mathrm{~m}, 8 \mathrm{H}, \mathrm{Ar}-\mathrm{H}), 4.33(\mathrm{q}, J=7.2$ $\left.\mathrm{Hz}, 2 \mathrm{H}, \mathrm{CH}_{2}\right), 2.62\left(\mathrm{~s}, 3 \mathrm{H}, \mathrm{CH}_{3}\right), 2.42\left(\mathrm{~s}, 3 \mathrm{H}, \mathrm{CH}_{3}\right), 1.35\left(\mathrm{t}, J=7.2 \mathrm{~Hz}, 3 \mathrm{H}, \mathrm{CH}_{3}\right) .{ }^{13} \mathrm{C}$ NMR $(100$ $\left.\mathrm{MHz}, \mathrm{CDCl}_{3}\right): \delta 13.6,14.2,21.3,60.9,100.7,111.1,117.4,121.4,124.9,128.0,128.5,129.4$, $130.2,130.3,134.2,139.7,148.7,151.3,154.4,156.7,157.6,161.8,162.6 . \mathrm{MS}(\mathrm{m} / \mathrm{z}, \%): 449$ $\left(\mathrm{M}^{+}, 15\right), 403$ (28), 311 (17), 283 (19), 159 (27), 133 (45), 91 (100). Anal. Calcd for $\mathrm{C}_{23} \mathrm{H}_{19} \mathrm{~N}_{3} \mathrm{O}_{7}$ (449.4): C, 61.47; H, 4.26; N, 9.35. Found: C, 61.65, H, 4.33; N, 9.25.

5-Ethoxycarbonyl-6-methyl-3-(3-methylphenyl)-2-(2-naphthaloxy)furo[2,3-d]pyrimidin4(3H)-one (6n). White crystals (yield 0.72 g, 79\%), m.p. $223-225{ }^{\circ} \mathrm{C}$. IR (KBr): $1728(\mathrm{C}=\mathrm{O})$, $1700(\mathrm{C}=\mathrm{O}), 1554,1294 \mathrm{~cm}^{-1} .{ }^{1} \mathrm{H}$ NMR $\left(400 \mathrm{MHz}, \mathrm{CDCl}_{3}\right): \delta 8.18-6.63(\mathrm{~m}, 11 \mathrm{H}, \mathrm{Ar}-\mathrm{H}), 4.34$ (q, $\left.J=7.2 \mathrm{~Hz}, 2 \mathrm{H}, \mathrm{OCH}_{2}\right), 2.53\left(\mathrm{~s}, 3 \mathrm{H}, \mathrm{CH}_{3}\right), 2.38\left(\mathrm{~s}, 3 \mathrm{H}, \mathrm{CH}_{3}\right), 1.32\left(\mathrm{t}, J=7.2 \mathrm{~Hz}, 3 \mathrm{H}, \mathrm{CH}_{3}\right)$.

${ }^{13} \mathrm{C} \mathrm{NMR}\left(100 \mathrm{MHz}, \mathrm{CDCl}_{3}\right): \delta 13.5,14.2,21.2,61.0,100.3,108.4,110.9,117.9,119.4,120.6$, $121.9,124.6$ (2), 125.2, 125.8, 126.3, 126.6, 127.3, 128.0, 128.5, 129.4, 130.1, 134.6, 139.7, 147.0, 155.2, 157.3, 162.6. MS (m/z, \%): $453\left(\mathrm{M}^{+}-1,100\right), 408$ (35), 311 (82), 283 (24). Anal. Calcd for $\mathrm{C}_{27} \mathrm{H}_{22} \mathrm{~N}_{2} \mathrm{O}_{5}$ (454.5): C, 71.36; H, 4.88; N, 6.16. Found: C, 71.15, H, 4.83; N, 6.09.

\section{Acknowledgements}

We gratefully acknowledge financial support of this work by the National Natural Science Foundation of China (No. 20772041) and Hubei Province (No. 2006ABB016), the Key Project of Chinese Ministry of Education (No. 107082) and Key Project of Hubei Provincial Department of Education (No. D200724001).

\section{References}

1. Shishoo, C. J.; Shirsath, V. S.; Rathod, I. S.; Yande, V. D. Eur. J. Med. Chem. 2000, 35, 351.

2. Kikuchi, H.; Yamamoto, K.; Horoiwa, S.; Hirai, S.; Kasahara, R.; Hariguchi, N.; Matsumoto, M.; Oshima, Y. J. Med. Chem. 2006, 49, 4698.

3. Duval, E.; Case, A.; Stein, R. L.; Cuny, G. D. Bioorg. Med. Chem. Lett. 2005, 15, 1885.

4. Santagati, M.; Modica, M.; Santagati, A.; Russo, F.; Spampinato, S. Pharmazie 1996, 51, 7.

5. Kidwai, M.; Rastogi, S.; Venkataramanan, R. Bull. Chem. Soc. Jpn. 2003, 76, 203.

6. Maruoka, H.; Yamagata, K.; Yamazaki, M. Liebigs Ann. Chem. 1994, 993. 
7. McGuigan, C.; Barucki, H.; Blewett, S.; Carangio, A.; Erichsen, J. T.; Andrei, G.; Snoeck, R.; De Clercq, E.; Balzarini, J. J. Med. Chem. 2000, 43, 4993.

8. McGuigan, C.; Yarnold, C. J.; Jones, G.; Vela'zquez, S.; Barucki, H.; Brancale, A.; Andrei, G.; Snoeck, R.; De Clercq, E.; Balzarini, J. J. Med. Chem. 1999, 42, 4479.

9. Teimouria, M. B.; Bazhrang, R. Bioorg. Med. Chem. Lett. 2006, 16, 3697.

10. Naya, S.; Tokunaka, T.; Nitta, M. J. Org. Chem. 2004, 69, 4732.

11. Zanatta, N.; Schneider, J. M. F. M.; Schneider, P. H.; Wouters, A. D.; Bonacorso, H. G.; Martins, M. A. P.; Wessjohann, L. A. J. Org. Chem. 2006, 71, 6996.

12. Chan, J.; Faul, M. Tetrahedron Lett. 2006, 47, 3361.

13. Palacios, F.; Alonso, C.; Aparicio, D.; Rubiales, G.; Santos, J. M. Tetrahedron 2007, 63, 523.

14. Brase, S.; Gil, C.; Knepper, K.; Zimmermann, V. Angew. Chem. Int. Ed. 2005, 44, 5188.

15. Palacios, F.; Herrán, E.; Alonso, C.; Rubiales, G. ARKIVOC 2007, (iv), 397.

16. López, J. L.; Tárraga, A.; Molina, P. ARKIVOC 2007, (iv), 39.

17. Ding, M. W.; Xu, S. Z.; Zhao, J. F. J. Org. Chem. 2004, 69, 8366.

18. Zhao, J. F.; Xie, C., Xu, S. Z.; Ding, M. W.; Xiao, W. J. Org. Biomol. Chem. 2006, 4, 130.

19. Yuan, J. Z.; Fu, B. Q.; Ding, M. W.; Yang, G. F. Eur. J. Org. Chem. 2006, 4170.

20. Li, H. X.; Xie, C.; Ding, M. W.; Liu, Z. M.; Yang, G. F. Synlett 2007, 2280.

21. Ding, M. W.; Yang, S. J.; Zhu, J. Synthesis 2004, 75.

22. Korte, F.; Trautner, K. Chem. Ber. 1962, 95, 307. 